9. Nolly, H., Carretero, O.A., Lama, M.C., Miatello, R., and Scicli, A.G. 1994. Vascular kallikrein in deoxycorticosterone acetate-salt hypertensive rats. Hypertension. 23(Suppl. I):I185-I188.

10. Nolly, H., Carretero, O.A., Scicli, G., Madeddu, P., and Scicli, A.G. 1990. A kallikrein-like enzyme in blood vessels of one-kidney, one-clip hypertensive rats. Hypertension. 16:436-440.

11. Carretero, O.A., and Scicli, A.G. 1995. The kallikrein-kinin system as a regulator of cardiovascular and renal function. In Hypertension: physiology, diagnosis, and management. J.H. Laragh and B.M. Brenner, editors. Raven Press. New York, New York, USA. 983-999.

12. Azizi, M., et al. 2005. Arterial and renal consequences of partial genetic deficiency in tissue kallikrein activity in humans. J. Clin. Invest. 115:780-787. doi:10.1172/JCI200523669.

13. Mulvany, M.J., et al. 1996. Vascular remodeling. Hypertension. 28:505-506.
14. Kaschina, E., et al. 2004. Genetic kininogen deficiency contributes to aortic aneurysm formation but not to atherosclerosis. Physiol. Genomics. 19:41-49.

15. Farhy, R.D., Carretero, O.A., Ho, K.-L., and Scicli, A.G. 1993. Role of kinins and nitric oxide in the effects of angiotensin converting enzyme inhibitors on neointima formation. Circ. Res. 72:1202-1210.

16. Ellenby, M.I., Ernst, C.B., Carretero, O.A., and Scicli, A.G. 1996. Role of nitric oxide in the effect of blood flow on neointima formation. J. Vasc. Surg. 23:314-322.

17. Meneton, P., et al. 2001. Cardiovascular abnormalities with normal blood pressure in tissue kallikrein-deficient mice. Proc. Natl. Acad. Sci. U. S. A. 98:2634-2639.

18. Bergaya, S., et al. 2001. Decreased flow-dependent dilation in carotid arteries of tissue kallikreinknockout mice. Circ. Res. 88:593-599.

19. Bergaya, S., Matrougui, K., Meneton, P., Henrion, D., and Boulanger, C.M. 2004. Role of tissue kallikrein in response to flow in mouse resistance arteries. J. Hypertens. 22:745-750.

20. Bakker, E.N.T.P., et al. 2002. Inward remodeling follows chronic vasoconstriction in isolated resistance arteries. J. Vasc. Res. 39:12-20.

21. Tomiyama, H., Scicli, A.G., Scicli, G.M., and Carretero, O.A. 1990. Renal effects of Fab fragments of kinin antibodies on deoxycorticosterone acetate-salt-treated rats. Hypertension. 15:761-766.

22. Fenoy, F.J., Scicli, A.G., Carretero, O., and Roman, R.J. 1991. Effect of an angiotensin II and a kinin receptor antagonist on the renal hemodynamic response to captopril. Hypertension. 17:1038-1044.

23. Saitoh, S., Scicli, A.G., Peterson, E., and Carretero, O.A. 1995. Effect of inhibiting renal kallikrein on prostaglandin $\mathrm{E}_{2}$, water, and sodium excretion. Hypertension. 25:1008-1013.

24. Alfie, M.E., Sigmon, D.H., Pomposiello, S.I., and Carretero, O.A. 1997. Effect of high salt intake in mutant mice lacking bradykinin- $\mathrm{B}_{2}$ receptors. Hypertension. 29:483-487.

\title{
Licking latency with licorice
}

\author{
Jeffrey I. Cohen
}

\author{
Medical Virology Section, Laboratory of Clinical Infectious Diseases, NIH, Bethesda, Maryland, USA.
}

\begin{abstract}
Numerous viruses cause latent infections in humans, and reactivation often results in pain and suffering. While vaccines for several of these viruses are available or currently being studied in clinical trials, and antiviral therapies have been successful in preventing or treating active infection, therapy to eradicate latent infection has lagged behind. A new study reported in this issue of the JCI shows that treatment of cells latently infected with Kaposi sarcoma-associated herpesvirus (KSHV) with glycyrrhizic acid, a component of licorice, reduces synthesis of a viral latency protein and induces apoptosis of infected cells (see the related article beginning on page 642). This finding suggests a novel way to interrupt latency.
\end{abstract}

Licorice, derived from the root of Glycyrrbiza glabra, has been used for more than 4 millennia as a flavoring agent in foods, beverages, and tobacco (1). Licorice is also used as an alternative medicine for the treatment of gastric and duodenal ulcers, sore throat, bronchitis, cough, arthritis, adrenal insufficiency, and allergic diseases. The licorice root contains numerous compounds, including glycyrrhizic acid (GA). It is estimated that in the United States, $3.3 \mathrm{mg}$ of GA is consumed per person daily. GA inhibits the replication of several viruses in vitro including herpesviruses, HIV, and the SARS coronavirus. When taken orally, GA is hydrolyzed to glycyrrhetic

Nonstandard abbreviations used: EBV, Epstein-Barr virus; GA, glycyrrhizic acid; KSHV, Kaposi sarcomaassociated herpesvirus; IL-6R, IL-6 receptor; LANA-1, latency-associated nuclear antigen 1 ; PEL, primary effusion lymphoma; Rb, retinoblastoma tumor-suppressor protein; v-cyclin, KSHV cyclin protein; $v$ FLIP, viral FLICE-inhibitory protein; vIL-6, viral IL-6.

Conflict of interest: The author has declared that no conflict of interest exists.

Citation for this article: J. Clin. Invest. 115:591-593 (2005). doi:10.1172/JCI200524507. acid by bacteria in the gastrointestinal tract before GA can be absorbed. Therefore, in Asia, where GA is used for the treatment of chronic hepatitis $\mathrm{B}$ or $\mathrm{C}$ infection, the drug is infused intravenously to achieve the appropriate serum levels.

In this issue of the JCI, Curreli et al. (2) show that GA induces apoptosis of primary effusion lymphoma (PEL) cells that are transformed by Kaposi sarcoma-associated herpesvirus (KSHV). KSHV is the etiologic agent of Kaposi sarcoma, and the virus is present in lesions from patients with multicentric Castleman disease and PEL. The latter presents as a malignant effusion located in the pleural, peritoneal, or pericardial space; tumor cells can also infiltrate the adjacent tissues. The virus is latent in PEL cells, which express a very limited set of viral proteins. The median survival time after diagnosis for patients with PEL is 6 to 12 months with chemotherapy and radiation therapy; thus, newer approaches to therapy are needed.

Curreli et al. (2) found that GA downregulates synthesis of the KSHV latency- associated nuclear antigen 1 (LANA-1) (Figure 1). LANA-1 is expressed in all KSHV-infected cells, including PEL cells. This protein allows the viral genome to be maintained as an episome in latently infected cells. LANA-1 binds to p53, inhibiting p53-mediated apoptosis, and interacts with the retinoblastoma tumor-suppressor protein $(\mathrm{Rb})$, which may prevent Rb-mediated cell cycle arrest. Curreli et al. found that downregulation of LANA-1 by GA was associated with an alteration in the mitochondrial membrane potential with translocation of apoptosis-inducing factor to the nucleus, DNA fragmentation, and apoptosis (2). In addition, cells treated with GA showed higher levels of phosphorylated (active) p53, which resulted in cell cycle arrest at the G1 checkpoint. GA upregulated expression of the KSHV cyclin protein ( $\mathrm{v}$-cyclin) but did not affect expression of the viral FLICEinhibitory protein (vFLIP). V-cyclin binds to and activates cyclin-dependent kinase 6 , which results in phosphorylation and inactivation of p53 and Rb. The increased level of v-cyclin in PEL cells treated with GA might also contribute to cell death, since overproduction of the protein has been reported to induce apoptosis.

\section{Additional molecular approaches to killing latent KSHV-infected PEL cells}

Other approaches have been considered for the treatment of PEL, based on KSHV gene expression in these tumors. Tumor cells 


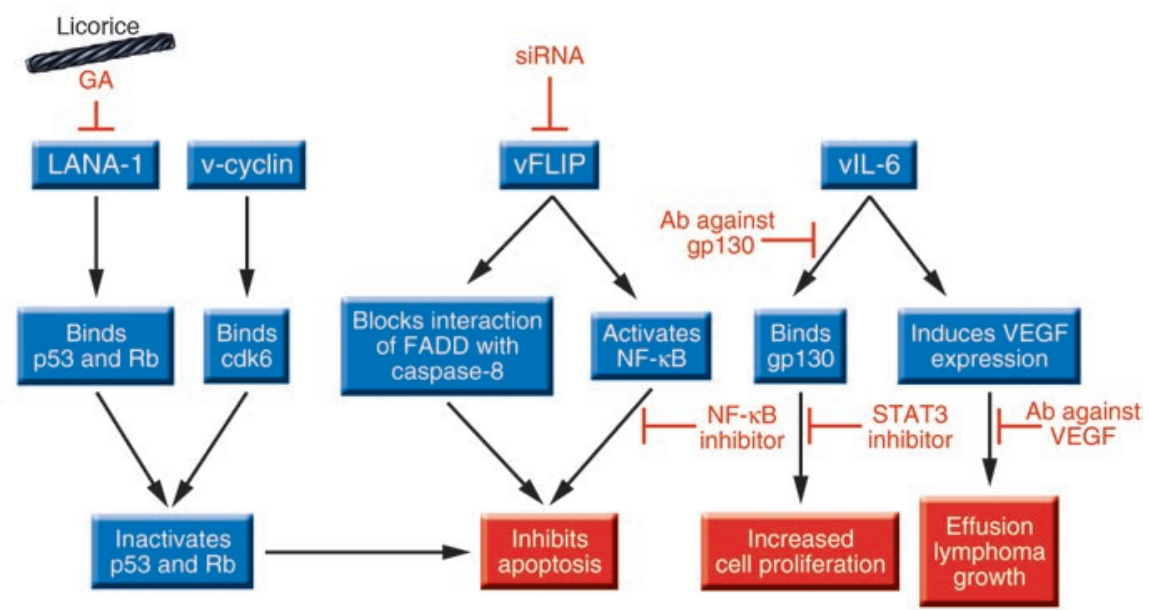

from patients with PEL produce several KSHV latency proteins, including LANA-1, v-cyclin, vFLIP, and viral IL-6 (vIL-6) (Figure 1) (3-5). vFLIP inhibits Fas-induced apoptosis and is responsible for most of the activation of NF- $\mathrm{KB}$ in PEL cells. Prevention of vFLIP production in PEL cells by transfection with small interfering RNA inhibited NF- $\kappa \mathrm{B}$ activity and induced apoptosis of the cells $(6,7)$. In addition, treatment of PEL cells with a small molecule that inhibits NF-кB (Bay 11-7082) induced apoptosis of these cells (8).

PEL cells synthesize vIL- 6 in vivo, and PEL cells secrete human IL- 6 and IL-10 and vIL- 6 in vitro. vIL- 6 functions as an autocrine growth factor for PEL cells in vitro, and the protein interacts with the signal transduction protein gp130 but not with the human IL-6 receptor (IL-6R). The interaction of vIL- 6 with gp130 leads to phosphorylation of STAT3, activation of the JAK-STAT pathway, and cell proliferation. Inhibition of STAT3 signaling by transfection of PEL cells with a dominant negative form of STAT3, or treatment of cells with a drug (AG490) that inhibits JAK2, resulted in decreased production of survivin and apoptosis of the cells (9). Other approaches have been used to inhibit the activity of IL-6. Antibody against gp130 or soluble IL-6R inhibited PEL cell growth in vitro (10). In another study, antibody against IL- 6 inhibited growth of PEL cell tumors in SCID mice (11). Antibody against human IL-10, and to a lesser extent antibody against vIL-6, inhibited growth of PEL cells in vitro (12). vIL-6 induces expression of VEGF, which promotes angiogenesis. PEL cells secrete high levels of VEGF and have VEGF receptors on their surface (13). Administration of neutralizing antibody against VEGF prevented development of effusion lymphomas in irradiated SCID/beige mice injected intraperitoneally with PEL cells.

\section{Targeted attack on latency of another human herpesvirus: Epstein-Barr virus}

Several approaches have been used to interrupt latency of Epstein-Barr virus (EBV), the other human $\gamma$-herpesvirus, which like KSHV infects and transforms B cells. Hydroxyurea eliminated EBV episomes from EBV-positive Burkitt lymphoma cells and reduced their malignant phenotype in an animal model (14). Low-dose hydroxyurea reduced the tumor size of EBV-positive central nervous system lymphomas in 2 patients with HIV (15).

Treatment of B cells latently infected with EBV with arginine butyrate (16) or gemcitabine (17) followed by ganciclovir induced viral replication and expression of the viral thymidine kinase, resulting in phosphorylation of ganciclovir and cell death in vitro. Infusions of EBV-specific cytotoxic $\mathrm{T}$ cells have been used successfully to treat patients with EBV lymphoproliferative disease (18) or EBVpositive Hodgkin disease (19), both of which are due to tumor cells latently infected with virus. Such approaches might also be tried for the treatment of patients with KSHV-positive PEL.

\section{Future considerations for GA therapy}

While GA is effective for killing PEL cells in vitro, there are several caveats for the treatment of patients with PEL with GA. First, since GA is rapidly hydrolyzed to glycyrrhetic acid in the gastrointestinal

\section{Figure 1}

Mechanisms of action of KSHV latency proteins expressed in PEL cells and points of attack by inhibitor compounds. cdk6, cyclindependent kinase 6; FADD, Fas-associated death domain-containing protein; siRNA, small interfering RNA. tract, glycyrrhetic acid would need to be shown to be effective against PEL cells in vitro or GA would need to be administered intravenously. After intravenous administration of GA for treatment of hepatitis, serum levels of GA have been shown to range from 40 to $100 \mu \mathrm{g} / \mathrm{ml}$ (20), compared with the millimolar concentrations needed to induce apoptosis of PEL cells in vitro. Thus, the levels of GA required for efficacy in vitro might not be achievable in vivo. Second, Curreli et al. (2) found that the effects of GA on downregulating LANA expression in PEL cells were reversible for up to 3-4 days of treatment; thus, continuous and/or prolonged courses of therapy with GA might be needed. Third, therapeutic levels of GA might be toxic for normal cells and tissues. Curreli et al. found that the $\mathrm{ED}_{50}$ of GA for PEL cells was 2-3 mM; however, levels of 5-6 mM were toxic for uninfected cells. Thus the therapeutic index for the treatment of PEL is likely to be low. Finally, it is not clear whether the effects of GA on PEL cell lines in vitro would also occur in tumors in vivo. Since intraperitoneal inoculation of immunodeficient mice with PEL cells results in malignant ascites, intravenous infusions of GA could be tested for efficacy in this model.

While a compound present in licorice may seem like an unlikely candidate for the treatment of virus-associated cancers, it is important to remember that other traditional drugs have proved highly effective for some infectious diseases. Extracts of the wormwood plant, which is a traditional Chinese medication for treatment of febrile illnesses, contain artemisinin; derivatives of this compound have become first-line treatments for drug-resistant 
malaria. Thus, derivatives of GA or other traditional medicines might be used in the future for treating human diseases caused by latent virus infections.

Address correspondence to: Jeffrey I. Cohen, Medical Virology Section, Laboratory of Clinical Infectious Diseases, Building 10, Room 11N228, NIH, 10 Center Drive, MSC 1888, Bethesda, Maryland 20892, USA. Phone: (301) 496-5265; Fax: (301) 496-7383; E-mail: jcohen@niaid.nih.gov.

1. Ploeger, B., et al. 2001. The pharmacokinetics of glyc yrrhizic acid evaluated by physiologically based pharmacokinetic modeling. Drug Metab. Rev. 33:125-147.

2. Curreli, F., Friedman-Kien, A.E., and Flore, O. 2005 Glycyrrhizic acid alters Kaposi sarcoma-associated herpesvirus latency, triggering p53-mediated apoptosis in transformed B lymphocytes. J. Clin. Invest. 115:642-652. doi:10.1172/JCI200523334.

3. Katano, H., Sato, Y., Kurata, T., Mori, S, and Sata, T. 2000. Expression and localization of human herpesvirus 8-encoded proteins in primary effusion lymphoma, Kaposi's sarcoma, and multicentric Castleman's disease. Virology. 269:335-344.

4. Parravicini, C., et al. 2000. Differential viral protein expression in Kaposi's sarcoma-associated herpesvirus-infected diseases: Kaposi's sarcoma, primary effusion lymphoma, and multicentric Castleman's disease. Am. J. Pathol. 156:743-749.

5. Moore, P, and Chang, Y. 2001. Kaposi's sarcomaassociated herpesvirus. In Fields virology. D.M. Knipe and P.M. Howley, editors. Lippincott Williams \& Wilkins. Philadelphia, Pennsylvania, USA. 2803-2833.

6. Guasparri, I., Keller, S.A., and Cesarman, E. 2004 KSHV vFLIP is essential for the survival of infected lymphoma cells. J. Exp. Med. 199:993-1003.

7. Matta, H., and Chaudhary, P.M. 2004. Activation of alternative NF-kappa B pathway by human herpes virus 8-encoded Fas-associated death domain-like IL-1 beta-converting enzyme inhibitory protein (vFLIP). Proc. Natl. Acad. Sci. U. S. A. 101:9399-9404.

8. Keller, S.A., Schattner, E.J., and Cesarman, E. 2000 Inhibition of NF-кB induces apoptosis of KSHVinfected primary effusion lymphoma cells. Blood. 96:2537-2542.

9. Aoki, Y., Feldman, G.M., and Tosato, G. 2003. Inhibition of STAT3 signaling induces apoptosis and decreases surviving expression in primary effusion lymphoma. Blood. 101:1535-1542.

10. Drexler, H.G., Meyer, C., Gaidano, G., and Carbone, A. 1999. Constitutive cytokine production by primary effusion (body cavity-based) lymphomaderived cell lines. Lenkemia. 13:634-640.

11. Foussat, A., et al. 1999. Human interleukin-6 is in vivo an autocrine growth factor for human herpesvirus-8-infected malignant B lymphocytes. Eur. Cytokine Netw. 10:501-508.

12. Jones, K.D., et al. 1999. Involvement of interleukin10 (IL-10) and viral IL-6 in the spontaneous growth of Kaposi's sarcoma herpesvirus-associated infected primary effusion lymphoma cells.
Blood. 94:2871-2879.

13. Aoki, Y., and Tosato, G. 1999. Role of vascular endothelial growth factor/vascular permeability factor in the pathogenesis of Kaposi's sarcomaassociated herpesvirus-infected primary effusion lymphomas. Blood. 94:4247-4254.

14. Chodosh, J., et al. 1998. Eradication of latent Epstein-Barr virus by hydroxyurea alters the growth-transformed cell phenotype. J. Infect. Dis. 177:1194-1201.

15. Slobod, K.S., et al. 2000. Epstein-Barr virus-targeted therapy for AIDS-related primary lymphoma of the central nervous system. Lancet. 356:1493-1494.

16. Mentzer, S.J., Perrine, S.P., and Faller, D.V. 2001. Epstein--Barr virus post-transplant lymphoproliferative disease and virus-specific therapy: pharmacological re-activation of viral target genes with arginine butyrate. Transpl. Infect. Dis. 3:177-185.

17. Feng, W.H., Hong, G., Delecluse, H.J., and Kenney, S.C. 2004. Lytic induction therapy for EpsteinBarr virus-positive B-cell lymphomas. J. Virol. 78:1893-1902.

18. Gottschalk, S., Rooney, C.M., and Heslop, H.E. 2005. Post-transplant lymphoproliferative disorders. Ann. Rev. Med. 56:29-44.

19. Bollard, C.M., et al. 2004. Cytotoxic T lymphocyte therapy for Epstein-Barr Virus+ Hodgkin's disease. J. Exp. Med. 200:1623-1633.

20. Van Rossum, T.G.J., Vulto, A.G., Hop, W.C.J., and Schalm, S.W. 1999. Pharmacokinetics of intravenous glycyrrhizin after single and multiple doses in patients with chronic hepatitis $\mathrm{C}$ infection. Clin. Ther. 21:2080-2090.

\title{
Unlocking the DEAD-box: a key to cryptococcal virulence?
}

\author{
Lena J. Heung' and Maurizio Del Poeta ${ }^{1,2}$
}

${ }^{1}$ Departments of Biochemistry and Molecular Biology, and 2Microbiology and Immunology, Medical University of South Carolina, Charleston, South Carolina, USA.

\begin{abstract}
The DEAD-box RNA helicases are enzymes involved in many critical aspects of RNA metabolism within both eukaryotic and prokaryotic organisms. Several studies have shown that these proteins may have important functions in mediating microbial pathogenesis. A new study in this issue of the JCI identifies the first DEAD-box RNA helicase in the pathogenic fungus Cryptococcus neoformans and proposes novel roles for this family of proteins in the development and progression of cryptococcosis (see the related article beginning on page 632 ).
\end{abstract}

Cryptococcosis is a chronic human disease caused by the ubiquitous environmental fungus Cryptococcus neoformans. The disease occurs after inhalation of yeast cells or basidiospores into the alveo-

Nonstandard abbreviations used: Not, negative on TATA-less; RNAi, RNA interference; SF, superfamily; Vad1, virulence-associated DEAD-box RNA helicaseencoding protein.

Conflict of interest: The authors have declared that no conflict of interest exists.

Citation for this article: J. Clin. Invest. 115:593-595 (2005). doi:10.1172/JCI200524508. lar spaces and eventually progresses with the dissemination of $C$. neoformans to the central nervous system, causing meningoencephalitis (1). The majority of cryptococcosis cases have been reported in immunocompromised patients, such as subjects with AIDS or those undergoing transplantation, but certain varieties of C. neoformans do affect immunocompetent hosts (1). Current therapies cannot completely eradicate the chronic infection, which necessitates life-long treatment. Therefore, studies addressing the under- standing of pathophysiological processes leading to the development of the disease are particularly important for the discovery of new therapeutic strategies. C. neoformans is a facultative intracellular pathogen with several well-established virulence factors, including growth at $37^{\circ} \mathrm{C}$ (temperature of the mammalian host), a large antiphagocytic polysaccharide capsule, and the laccase enzyme, which can produce melanin pigments from host-derived substrates. With the goal of identifying novel targets for drug development, current Cryptococcus research is focused on the signaling networks that regulate these virulence factors. A few virulence-related pathways have been identified in C. neoformans, including the $\mathrm{G} \alpha$ protein-cAMP-PKA and Ipc1-Pkc1 pathways $(2,3)$. In this issue of the JCI, Panepinto et al. (4) describe a new class of proteins and a novel signaling net- 